\title{
Model Based Control Strategies for a Class of Nonlinear Mechanical Sub-Systems
}

\author{
Clara M. IONESCU and Robin DE KEYSER \\ Ghent University, Department of Electrical energy, Systems and Automation, Research group on Dynamical Systems and Control \\ Technologiepark 914, 2nd floor, 9052 Ghent, Belgium Email: claramihaela.ionescu@UGent.be
}

\begin{abstract}
This paper presents a comparison between various control strategies for a class of mechanical actuators common in heavy-duty industry. Typical actuator components are hydraulic or pneumatic elements with static non-linearities, which are commonly referred to as Hammerstein systems. Such static non-linearities may vary in time as a function of the load and hence classical inverse-model based control strategies may deliver sub-optimal performance. This paper investigates the ability of advanced model based control strategies to satisfy a tolerance interval for position error values, overshoot and settling time specifications. Due to the presence of static non-linearity requiring changing direction of movement, control effort is also evaluated in terms of zero crossing frequency (up-down or leftright movement). Simulation and experimental data from a lab setup suggest that sliding mode control is able to improve global performance parameters.
\end{abstract}

Index Terms-sliding mode control, nonlinear system, static nonlinearity, internal model control, adaptive gain

\section{INTRODUCTION}

A class of mechanical systems with nonlinear characteristics are found in automotive industry. To meet future requirements on automotive systems in terms of increased engine power, while reducing fuel consumption and pollutant emissions, new camless engines are required [1]. One of the most common solutions is an independent valve actuator system able to realize Variable Valve Actuation (VVA) operations. To this aim, a promising solution relies on Electromechanical Valve Actuators (EMVA) technology, where an electronic control system commands each valve properly at every engine speed [2]. The effectiveness of the EMVA system depends on the performances of the control strategies necessary to guarantee precise valve closing/opening. The control must account for the behaviour of the system which is strongly affected by many nonlinearities such as friction, motion constraints, delays, etc.

Another class of position controlled mechanical actuators are encountered in agricultural machines. Of the many agricultural machines available in today's mechatronic applications, the harvesting machines are the most difficult to automatize due to the presence of nonlinear dynamics, time delay, uncertainties in model parameters and interaction with the environment (i.e. crop dynamic environment) [3], [4]. A special class of harvesting machines are those composed of tilting and rotating mechanisms for crop harvesting and/or processing. Main types are: i) grain harvesting (tilting the header for distance to the ground regulation) and ii) forage harvesters (rotating the spout for angle of crop flow to a reservoir), both for position control applications.

Challenges for control are numerous, since these complex mechanical systems are inter-coupled with sub-systems delivering pressure, flow or current and which in turn depend on the net power available in the machine. The required power may change in time due to environmental conditions (slope, crop density, speed of harvesting, etc) [5]. Modelling based on physical/mechanical/electrical principles is nearly impossible and thus black box modelling is usually performed via identification techniques to obtain a simplified model of the sub-system and design control strategies.

In this paper we investigate the application of model based control systems to position control of a mechanical system with input-output slew rates, saturation, time delay and static nonlinearity. The system is quite challenging for control. It contains time delay combined with very fast dynamics in the actuators (motor), hence it can be approximated by a delayed integrator.

The paper is organized as follows: the next section presents the various parts of the system to be controlled. Sections III and IV introduce the control strategies and the corresponding controller design parameters. Section $\mathrm{V}$ presents the result in simulation and on real experimental setup. A conclusion section summarizes the main outcome of this work.

\section{A Class of Hammerstein Models}

Actuator solutions in heavy-duty industrial applications where torque and force may be required at high speed and in varying time conditions are usually hydraulic-based elements. The hydraulic circuit is mostly controlled by means of proportional servo valve (PSV) which may be pressure dependent. In order to make things easier from point of view of dynamic compensation of nonlinear effects, one may attempt to make this PSV pressure independent by using a pressure compensated flow restriction valve. The flow usually feeds a gearotor motor transforming it into rotation movement. The modelling difficulties for control purposes are the large range of operating points, fast system dynamics and the lack of sensors in such subsystems.

Depending on the load and its corresponding force a safety mechanism based on spring-damper elements prevents the motor from overload. In the practical operating range, this mechanism will absorb most of the influence of the inertia 
and some backlash in the gearing. As such, friction is the main origin of torque on the engine and dynamical influences are difficult to model.

In the literature, a number of modelling strategies for hydraulic systems are proposed. The most common way to model the kind of systems discussed in this paper is using greybox non-linear models [6], [7], [8], based on basic hydraulic principles described in [9] or via bondgraphs [10]. The systems discussed in these papers are academic setups with a high number of precise sensors (pressure sensors, spool position sensors) required for fast and precise control, e.g. in active suspension systems. This makes identification of the separate subsystems and system control a lot easier compared to reallife situations. An overview of linear and nonlinear modelling techniques can be found in [11], [12]. Here, the comparison between linear models, neural networks and wavelet networks and their different approaches is being made. As the latter two methods are computational heavy, no good results were achieved due to the lack of sensor precision, and basic (nonlinear) models are required for the subsequently discussed control strategies, one may consider a simplified model.

A simple representation of such mechanical systems is the Hammerstein model structure. For this, the static nonlinearity needs to be defined. The main non-linear behaviour of the system is exerted in the hydraulic part. The dynamics are rather fast, hence they can be ignored in this part of the subsystem. Typical non-linearities which may be found in such mechanical systems are dead zone, saturation, mechanical limits etc. Figure 1 depicts the static characteristic present in our system as available from experimental data. The various colours depict the changes of the operating range, as a result of different load conditions (e.g speed and type of product).

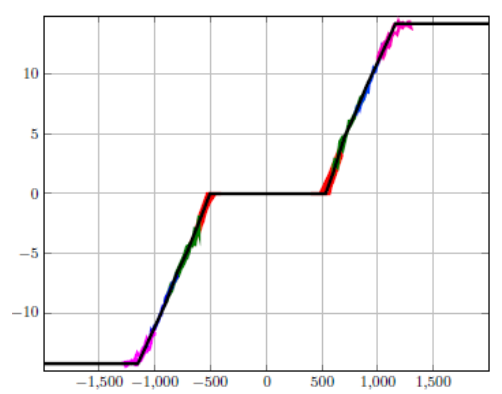

Fig. 1. Static characteristic of the nonlinearity present in the system; notice that the slope may vary depending on the operating conditions, i.e. crop speed and volume per time unit. Arbitrary units: $\mathrm{X}$-axis current and Y-axis flow.

Another source of difficulty in closed loop control operation is the presence of time-delays. The delay mainly depends on the hydraulic system, more exactly on the spool position and pressure build-up in the tubing in the system. Because of the lack of precision in the angle sensor, it is very difficult to define the transition between the delay and the beginning of the dynamics.

Delay estimation techniques are numerous and vary from simple signal processing cross-correlation algorithms [13] to

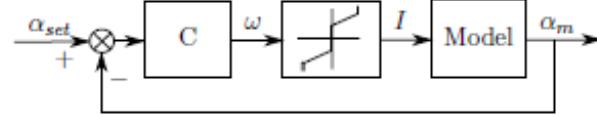

Fig. 2. Default closed loop control scheme for position control.

more complex time varying window estimation algorithms [14], [15]. Delay compensators can be used but they require complex control architectures [16]. In our situation, we assumed the delay is fixed to a certain value and used it directly in the controller design, assuming a robust controller.

If one integrates the above into a Hammerstein-model, one obtains a static non-linear characteristic with linear dynamics, with time delay. The universal controller module (UCM) and the sensor represent the quantization in the system. We assume that the angle sensor has a limited precision, i.e. introduces an output quantization of $0.75^{\circ}$. As the current is sent through the controller area network (CAN)-bus to the UCM, also an input quantization of $10 \mathrm{~mA}$ is present in the system.

To summarize, the dynamic part of the following blocks after the static nonlinearity block in figure 2 can thus be represented by the following transfer function:

$$
\frac{\alpha(s)}{\omega(s)}=\exp ^{-0.009 s} \frac{1}{s}
$$

from angular velocity ( $\mathrm{rad} / \mathrm{s})$ to angle position (degrees).

\section{LiNEAR CONTROL STRATEGIES}

The default controller available for testing closed loop position control is a $\mathrm{P}$ controller with gain scheduling. Gain scheduling algorithms may prove quite effective when used with advanced control strategies [17]; however, due to the increased numerical complexity these cannot be used in this application. The $\mathrm{P}$ controller was tuned using trial an error. The controller output is converted to a current value using the inverted non-linear characteristic, as in figure 2 .

We will assume the same configuration for the subsequent control strategies and focus on the design of the C-block only. Lead, PI and PID controllers are taken in consideration, due to their simplicity and hence high implementation ability in modular communication systems available in industry. For tuning the controllers, we make use of the computer aided design tool developed in our lab and under Matlab use: FRTool [18].

The controller is implemented then in a microcontroller board which sends equivalent positive/negative values to the actuator. This is then evaluated in terms of zero-crossings (ZC) as an indicator of controller effort (aggressiveness). The results of these controllers have been reported in [19].

\section{Advanced Model Based Control Strategies}

\section{A. Internal Model Control with gain adaptation}

The next proposed control strategy is internal model control (IMC). The main philosophy behind this controller is to 
reverse, i.e. invert, the influence of the system on the reference signal $\alpha_{\text {set }}$, resulting in a perfectly tailing system output. Of course, there are certain issues with the practical implementation of the idea, mainly due to the modelling uncertainty. Adding an adaptive mechanism could cope with this problem [20], [21]. Further more, feedback for disturbance rejection and filtering to cope with the non proper compensator transfer function are added and only the invertible part is inverted.

In a first instance, the delay is neglected. Working in discrete time without delay, the process model $P$ is equal to the good part $P_{g}$, thus the bad part is $P_{b}=1$ :

$$
P\left(q^{-1}\right)=\frac{T_{s}}{1-q^{-1}}=P_{g}\left(q^{-1}\right)
$$

In order to ensure zero SS error, the following filter is used:

$$
F\left(q^{-1}\right)=\left(\frac{1+a}{1+a q^{-1}}\right)^{n}\left(1-f+f q^{-1}\right)
$$

where $a$ is the discrete time filter parameter, with $a=$ $-e^{-T s / \lambda}$, in which $\lambda$ is the continuous time (tunable) filter parameter. The value of $n$ is the difference between the order of the numerator and the denominator of $P_{g}$ plus one, i.e. $n=2$ in this case. Finally, $f$ is given by the following equation, in which $p_{n}$ is the coefficient of the $n$-th order term of the bad part of the numerator of $P$ :

$$
f=\frac{n a}{1+a}-\sum_{i=1}^{m} i \cdot p_{i}
$$

The filter then becomes the following transfer function:

$$
F\left(q^{-1}\right)=\frac{(1+a)\left((1-a)+2 a q^{-1}\right)}{\left(1+a q^{-1}\right)^{2}}
$$

and this concludes the development of the classic IMC controller. The only remaining step is the tuning of the $\lambda$ parameter. An optimization procedure has been used to determine the value between $[0,1]$ and the solution of $0.39 \mathrm{~s}$ has been found to give minimum IAE. This then allows us to give values to the $a$ parameter in the above equations.

Since uncertainty in the static-characteristic is significant as this changes with operating conditions, we should introduce this in the IMC controller under the form of an extra parameter $G$ in the process transfer function, hence:

$$
P\left(q^{-1}\right)=\frac{G \cdot T_{s}}{1-q^{-1}}=P_{g}\left(q^{-1}\right)
$$

with the same filter as in (5). The controller is given by:

$$
C\left(q^{-1}\right)=\frac{P_{g}\left(q^{-1}\right) F\left(q^{-1}\right)}{1-P_{g}^{-1}\left(q^{-1}\right) F\left(q^{-1}\right) P\left(q^{-1}\right)}
$$

or equivalently

$$
C(z)=\left(\frac{1+a}{a^{2} G T_{s}}\right)\left[\frac{1+a}{T_{s}} \frac{T_{s} z}{z-1}-2 a\right]
$$

which is a PI controller tuned via IMC rules. We expect in this system the gain varies between 0.5 to 1.25 . This implies that:

$$
\frac{1+a}{a^{2} G T_{s}}=\frac{1+\hat{a}}{\hat{a}^{2} T_{s}}
$$

and the transfer function of the controller is updated with this term. Adaptation of the gain implies adaptation of the lambda value. Knowing the interval of variation we expect in the gain, we can also estimate an interval for the lambda value:

$$
\hat{\lambda}=-\frac{T_{s}}{\ln \left(1-\frac{1+a}{a^{2} G}\right)} \in[0.18 ; 0.46]
$$

We propose the following simple yet effective estimation algorithm:

$\hat{\lambda}_{k}=\left(1-f_{f}\right) \hat{\lambda}_{k-1}+g \int\left(\hat{\omega}_{m}-\omega_{w i s h}\right)^{2} d t-h \int\left(\alpha_{s e t}-\alpha_{m}\right)^{2} d t$

As $f_{f}$ is a small but positive value, $\hat{\lambda}$ will decrease over time, resulting in an increasing $\mathrm{K}$. This will render the controller more reactive and the closed loop system to be more oscillatory, increasing the IAE. However, these oscillations will be far more expressed in the speed $\omega$ [22]. If the integrated squared error (ISE) of $\hat{\omega}_{m}-\omega_{\text {wish }}$ increases, with $\omega_{\text {wish }}$ the controller output, then $\hat{\lambda}_{k}$ will increase to, rendering the system to be slower, as $\mathrm{K}$ decreases. This will result in a very slow controller, so the ISE of $\alpha_{s e t}-\alpha_{m}$ will increase, decreasing the value of $\hat{\lambda}_{k}$ and increasing $\mathrm{K}$. The main influence remains the ISE of the speeds, that is why the implementation can be presented as shown in figure 3.

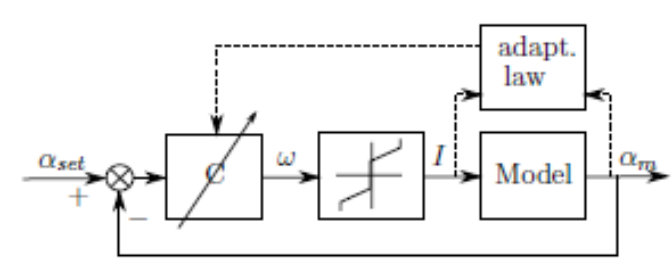

Fig. 3. Block Scheme of the Adaptive IMC control strategy.

Of course, there are some restrictions to be added to the system. First of all, $\hat{\lambda}_{k}$ should remain within the known bounds, and a maximum to the integrals should be posed, in order to cope with the disturbances in $\alpha_{\text {set }}$. Further more, (computational light) IIR-filters are used instead of integrals to reduce the effect of (corrected) errors on the long term. Next, thresholds are added in order to minimize the number of corrective actions of the integrals, so called conditional updating [21]. The velocity $\hat{\omega}_{m}$ is estimated using a Kalman filter. The parameters in (11) are chosen to be: $f=6 \cdot 10^{-5}$, $g=4 \cdot 10^{-6}$ and $h=1 \cdot 10^{-6}$.

\section{B. Sliding Mode Control}

Since all previously described controllers were designed for discrete time and in order to cope with the finite sampling, a discrete time SMC controller is designed [23], [24], [25]. Considering an uncertain first order discrete time linear system in which the following notation is introduced, i.e. $\Delta A=B \tilde{a}$, $\Delta B=B \tilde{b}, \mathbf{d}=B \tilde{d}$ :

$$
\begin{aligned}
\mathbf{x}_{k+1} & =(A+\Delta A) \mathbf{x}_{k}+(B+\Delta B) u_{k}+\mathbf{d}_{k} \\
& =A \mathbf{x}_{k}+B\left(u_{k}+f_{k}\right)
\end{aligned}
$$


where $f_{k}=\tilde{a} \mathbf{x}_{k}+\tilde{b} u_{k}+\tilde{d}_{k}$ is the generalized form of disturbance, containing all uncertainties in the system. First, assuming $f_{k}=0$ we have that:

$$
\begin{aligned}
s s_{k+1}=0 & =\sum \mathbf{e}_{k+1} \\
& =\sum A \mathbf{x}_{k}+\sum B u_{k}-\sum \mathbf{x}_{s e t, k+1} \\
u_{e q, k} & =\left(\sum B\right)^{-1}\left(\sum \mathbf{x}_{s e t, k+1}-\sum A \mathbf{x}_{k}\right)
\end{aligned}
$$

A switching law based on the following law $s i=$ $-\nu \operatorname{sign}(s s)-p \cdot s s$, with $\nu>0, p>0$ and where $-k \cdot s s$ is added for speed [23]. For the total control law we have

$$
\begin{aligned}
s s_{k+1} & =q \cdot s s_{k}-\nu \operatorname{sign}\left(s s_{k}\right)=\sum A \mathbf{x}_{k}+\sum B u_{k} \\
& -\sum \mathbf{x}_{s e t, k+1} \\
u_{e q, k} & =\left(\sum B\right)^{-1}\left(\sum \mathbf{x}_{s e t, k+1}-\sum A \mathbf{x}_{k}+s s_{k+1}\right)
\end{aligned}
$$

Stability analysis from [23] suggests the following conditions:

$$
\mid \begin{aligned}
& s s_{k} \mid \geq \sum B f_{\max }+\nu \rightarrow s s_{k+1}^{2}<s s_{k}^{2} \\
& \left|s s_{k}\right| \geq \sum B f_{\max }+\nu \rightarrow s s_{k+1}^{2}<\sum B f_{\max }+\nu
\end{aligned}
$$

where $\sum B f_{\max }<\nu$ with $\forall k \in \aleph:\left|f_{k}\right|<f_{\max }$. As such, convergence is guaranteed, but for large values of $f_{\max }$ the boundary layer to the sliding surface may be intolerably big, resulting in a very chattering behaviour. In order to reduce this and to improve overall performance a number of actions are taken:

- a disturbance observer is introduced as:

$$
\begin{aligned}
& u_{o b s, k}=-\hat{f}_{k}=-\hat{f}_{k-1}-\left(\sum B\right)^{-1} . \\
& \cdot g\left(s s_{k}-q \cdot s s_{k-1}+\nu \operatorname{sign}\left(s s_{k-1}\right)\right)
\end{aligned}
$$

- a recursive switching function is used:

$$
s s_{k}=\sum \mathbf{e}_{k}+\phi \cdot s s_{k-1}
$$

with $0<\phi<1$;

- to deal with time delay, the control vector $u_{e q}$ is shifted for the number of samples of delay (estimated here to 9 samples);

- chattering is reduced using a sigmoid function

$$
\operatorname{sigm}(x)=\frac{x}{|x|+\psi}
$$

by lowering the dead zone bounds of the static nonlinearity from current to speed.

The SMC is then implemented using the following definitions:

$$
\mathbf{x}=\left[\begin{array}{c}
\alpha \\
\omega
\end{array}\right], A=\left[\begin{array}{ll}
1 & 0 \\
0 & 0
\end{array}\right], B=\left[\begin{array}{c}
\frac{T_{s}}{G} \\
\frac{1}{G}
\end{array}\right]
$$

with $T_{s}$ the sampling period $(100 \mathrm{~Hz})$ and $G=38$ the inverse of the gain of the system, i.e. an estimation of the slope of the calibration characteristic. The controller parameter values are chosen to be as follows:

$$
\sigma=3 ; \phi=0.15 ; q=1 ; g=0.3 ; \psi=10
$$

The value of $\nu$ is kept as low as possible and formulated as:

$$
\nu=\frac{1+T_{s} \sigma}{G} \frac{m}{g}+\delta
$$

where $m>\left|f_{k}-f_{k-1}\right|, \forall k \in \aleph$ and $\delta$ an arbitrary small value of 0.01 . The controller is implemented as in figure 4, making use of the same velocity estimator as the IMC controller.

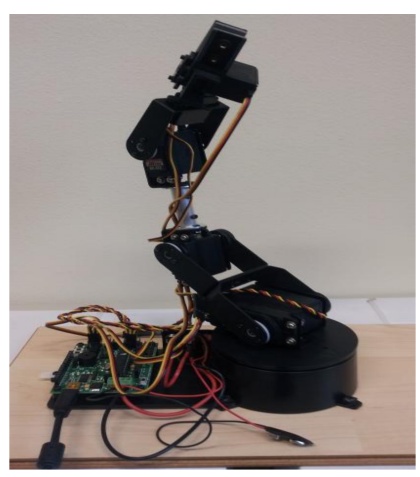

Fig. 5. A picture of the lab setup used to test the control strategies.

TABLE I

IAE IN DEGREES AND ZC IN FREQUENCY HZ

\begin{tabular}{cccc}
\hline & Ideal Case & Gain $-50 \%$ & Pole at $\mathrm{s}=-10$ \\
\hline Current & $0.86 / 2.1$ & $1.48 / 1.1$ & $0.91 / 1.6$ \\
IMC & $0.42 / 2.4$ & $0.49 / 1.4$ & $0.80 / 2.0$ \\
AIMC & $0.39 / 3.8$ & $0.48 / 2.1$ & $0.55 / 2.0$ \\
SMC & $0.48 / 1.4$ & $0.49 / 1.0$ & $0.52 / 1.5$ \\
\hline
\end{tabular}

\section{Results}

The control performance is evaluated on a specially designed setup, for a ramp with slope $9^{\circ} / s$ slope and inputoutput slew rates as in section II. The lab device is depicted in Figure 5. Three situations are envisaged:

- a perfectly known model (ideal situation);

- a drop in system gain of $-50 \%$ and

- an extra pole added to the system at $\mathrm{s}=-10$.

The changes in gain and added dynamics reflect changes in the static characteristic and in the velocity components of the system under various operating conditions. Typical operating signals from application field have been used to test the controllers: step response, ramp and tracking signals.

The closed loop performance of the model based advanced controllers versus the default one available in the system is given in figure 6 for the ideal case, in figures 7 for changes in the gain and for extra dynamics in the system with corresponding IAE and ZC values summarized in Table I.

The default controller (in figure legend denoted by 'current') has good response for step reference but has a significant SS error for ramp input. This error increases with gain variations and with additional dynamics in the loop. It also cannot cope with the tolerance interval in the error values ( \pm 2 degrees). On the other hand, it is obvious that model based advanced control techniques will deliver improved closed loop performance. Of all these controllers, SMC provides best performance in terms of low IAE with lowest frequency in ZC. Table II provides an overview of all controllers and their performance in all three cases tested.

\section{CONCLUSION}

This paper evaluated advanced model based control strategies for a class of mechanical actuator systems commonly 


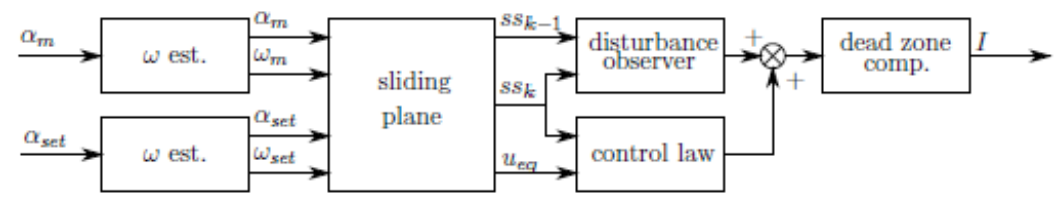

Fig. 4. Block Scheme of the SMC control strategy.
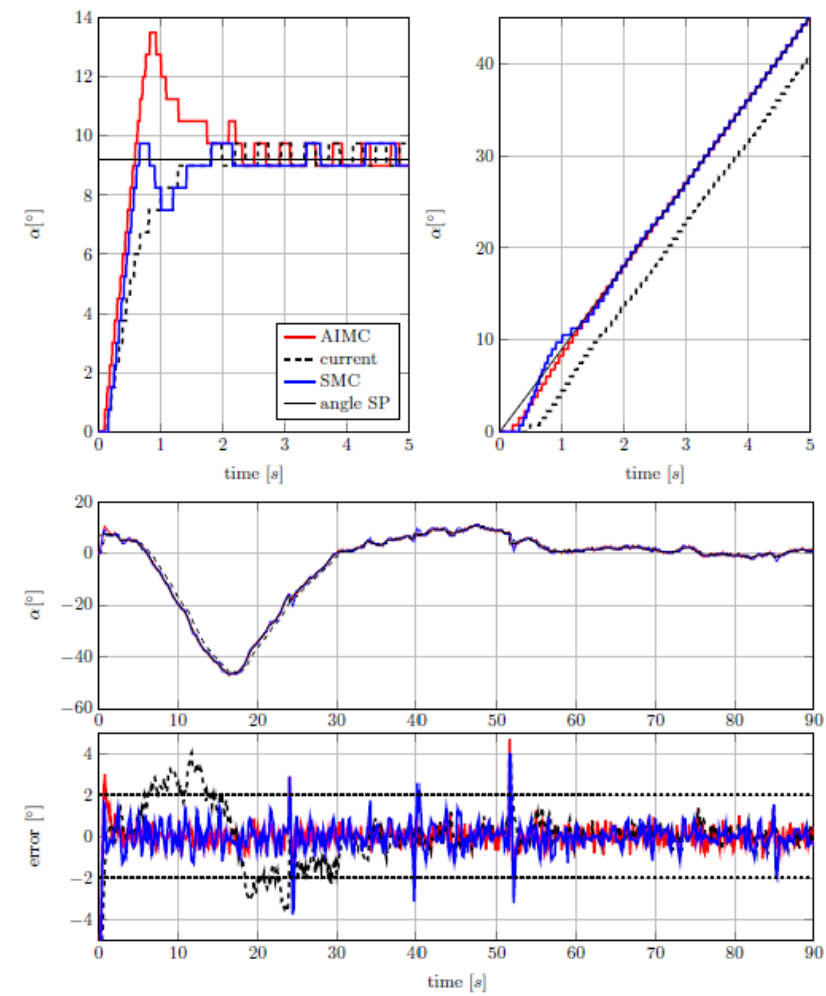

Fig. 6. Ideal situation: no modelling errors. Error tolerance between the two dashed lines. Simulation results for step and ramp setpoint; experimental data for tracking.

TABLE II

GLOBAL ASSESSMENT OF CLOSED LOOP PERFORMANCE

\begin{tabular}{ccccc}
\hline Controller & Error & SS-II & Robust & ZC \\
\hline default & + & - & \pm & + \\
IMC & + & + & - & + \\
AIMC & + & + & + & \pm \\
SMC & + & + & + & + \\
\hline
\end{tabular}

found in harvesting machines. Typically, these systems are classified as Hammerstein-type systems, with static nonlinear characteristics and fast dynamics. Additional dynamics as time-delays are introduced to mimic real life situations and closed loop performance is evaluated with slew rates on inputoutput variables.

Our results with simulated and real experimental data suggest that global requirements such as IAE and number of zerocrossings in the control effort (due to static nonlinearity) are well met by applying sliding mode control.

\section{REFERENCES}

[1] M. M. Schechter and M. B. Levin, "Camless engine", SAE Technical Paper (1996), no. 960581

[2] A. di Gaeta, V. Giglio, and G. Police, "Model-based decoupling control of a magnet engine valve actuator", SAE International Journal of Engines, 2,pp. 254-271, 2010

[3] W. Saeys, B. Lenaerts, G. Craessaerts, J. De Baerdemaeker, "Estimation of the crop density of small grains using LiDAR sensors", BIOSYSTEMS ENGINEERING 102(1), pp. 22-30, 2009

[4] M. Reyniers, K. Maertens, E. Vrindts, J. De Baerdemaeker, "Yield variability related to landscape properties of a loamy soil in central Belgium", SOIL \& TILLAGE RESEARCH, 88(1-2), pp. 262-273, 2006

[5] B. Lenaerts, T. Aertsen, E. Tijskens, B. De Ketelaere,H. Ramon, J. De Baerdemaeker, W. Saeys, "Simulation of grain-straw separation by Discrete Element Modeling with bendable straw particles", COMPUTERS AND ELECTRONICS IN AGRICULTURE, 101, pp. 24-33, 2014

[6] R. Poley, "DSP control of electro-hydraulic servo actuators", Texas Instruments, Tech. Rep., 2005.

[7] D. Maneetham, N. Afzulpurkar, "Modeling, simulation and control of high speed nonlinear hydraulic servo system", World Journal of Modelling and Simulation,6(1), pp. 27-39, 2010.

[8] B. Yao et al., "Adaptive robust motion control of single-rod hydraulic actuators: theory and experiments", IEEE/ASME Transactions on Mechatronics, 5(1), pp. 79-91, 2000.

[9] H. Merrit, Hydraulic Control System. John Wiley \& Son, inc., 1967. 

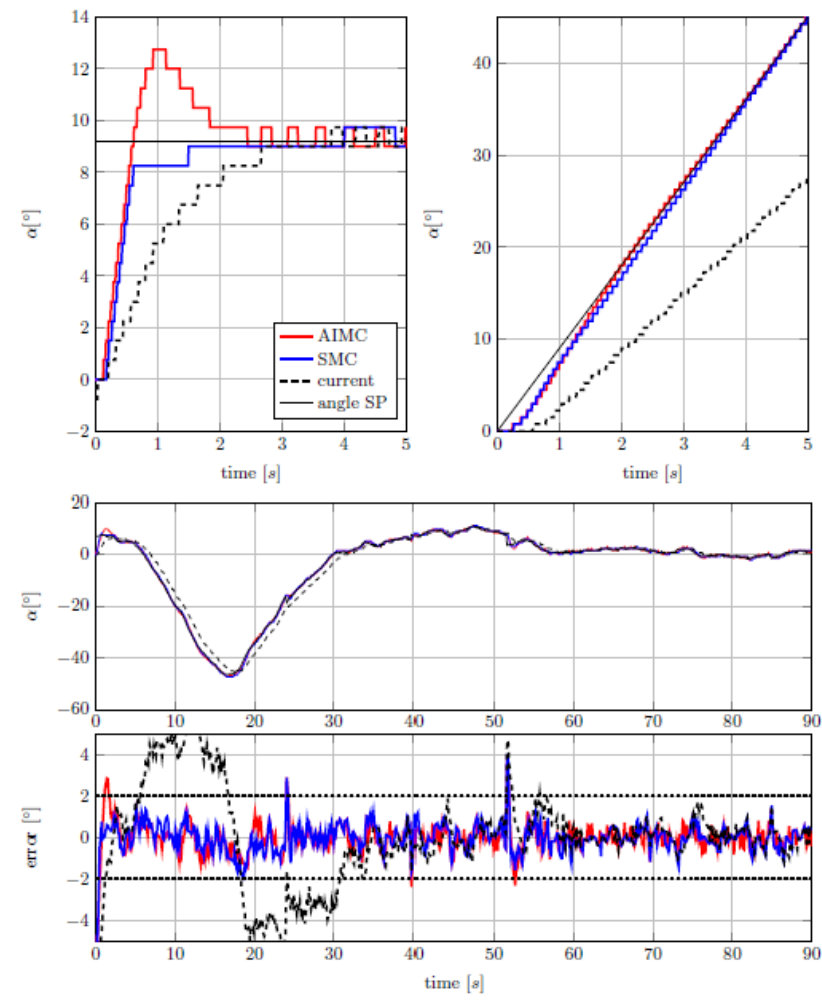
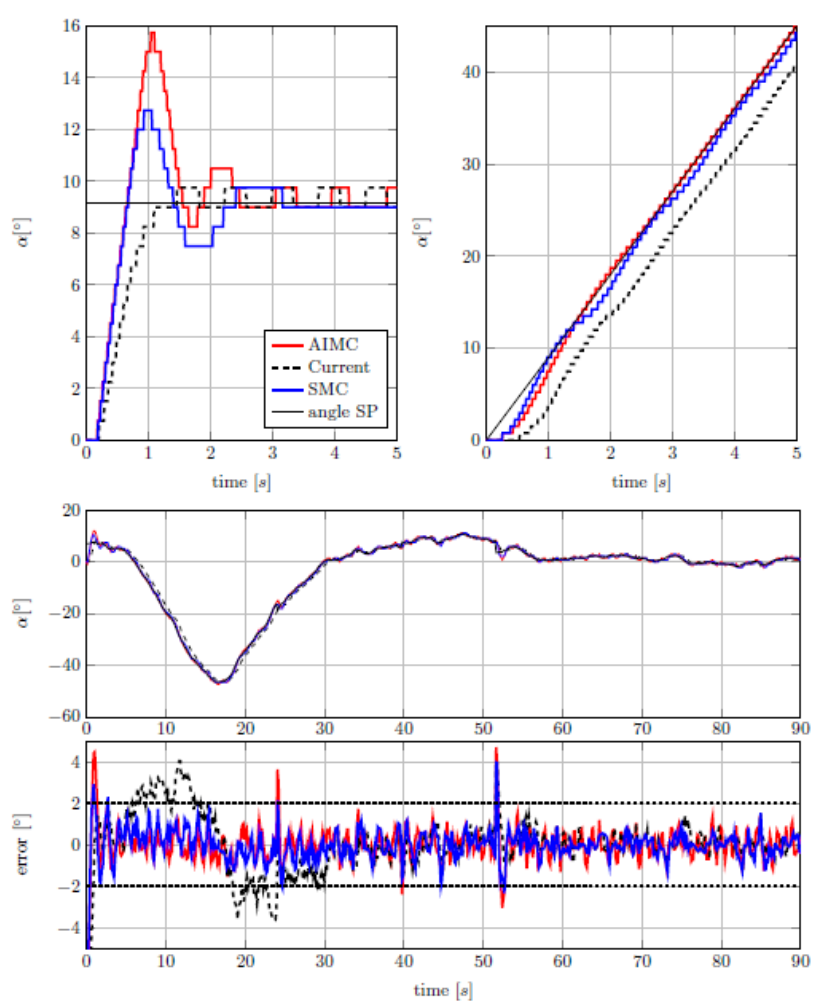

Fig. 7. Change in 50\% gain situation (left) and additional dynamics situation (right). Error tolerance between the two dashed lines. Simulation results for step and ramp setpoint; experimental data for tracking.

[10] K. Dasgupta, H. Murrenhofi, "Modelling and dynamics of a servo-valve controlled hydraulic motor by bondgraph", Mechanism and Machine Theory, 46, pp. 1016-1035, 2011.

[11] J. Sjoberg et al., "Nonlinear black-box modeling in system identification: a unified overview", Automatica, 31(12), pp. 1691-1724, 1995.

[12] I. Rivals , L. Personnaz, "Black-box modelling with state-space neural networks", Neural Adaptive Control Technology, pp. 237-264, 1996.

[13] C.M. Ionescu, R. Hodrea, R. De Keyser, "Variable Time-Delay Estimation for Anesthesia Control During Intensive Care", IEEE TRANSACTIONS ON BIOMEDICAL ENGINEERING, 58(2), pp. 363-369, 2011

[14] C. Pop, C.M. Ionescu, R. De Keyser, E.Dulf, " Robustness evaluation of fractional order control for varying time delay processes", SIGNAL IMAGE AND VIDEO PROCESSING, 6(3), Special Issue: SI, pp. 453461, 2012

[15] C. Pop, C.M. Ionescu, R. De Keyser, " Time delay compensation for the secondary processes in a multivariable carbon isotope separation unit", CHEMICAL ENGINEERING SCIENCE, 80, pp. 205-218, 2012

[16] M. Galvez-Carrillo, R. De Keyser, C.M. Ionescu, "Nonlinear predictive control with dead-time compensator: Application to a solar power plant", SOLAR ENERGY, 83(5), pp. 743-752, 2009

[17] J. Nino, R. De Keyser, S. Syafiie, C.Ionescu, M. Struys, 'EPSACcontrolled anesthesia with online gain adaptation", INTERNATIONAL JOURNAL OF ADAPTIVE CONTROL AND SIGNAL PROCESSING, 23(5), Special Issue: SI, pp. 455-471, 2009

[18] R. De Keyser, C.M. Ionescu, "FRtool: a frequency response tool for CACSD in Matlab", IEEE Conf. on Computer Aided Control Systems Design, Munich, pp. 2275-2280, 2006.

[19] C.M. Ionescu, R. De Keyser, "Comparison of linear control algorithms for a class of nonlinear mechanical actuators", Proc of the 19th International Conference on System Theory, Control and Computing (ICSTCC), October 14-16, Cheile Gradistei, Romania, 372-377, 2015

[20] F. Yu, ZZ. Mao, MX. Jia, "Recursive identification for HammersteinWiener systems with dead-zone input nonlinearity", J. Proc Ctrl, 23(8), 1108-1115, 2013.

[21] K. J. Åström, B. Wittenmark, Adaptive Control, Dover edition. AddisonWesley Publishing Company, Inc., 2008
[22] R. J. E. Merry, M. van de Molengraft, M. Steinbuch, "Velocity and acceleration estimation for optical incremental encoders", Mechatronics, 20, 20-26, 2010

[23] J. Liu, X. Wang, Advanced Sliding Mode Control for Mechanical Systems, Springer, 2012

[24] B.-C. Zheng, G.-H. Yang, "Quantized output feedback stabilization of uncertain systems with input nonlinearities via sliding mode control", International Journal of Robust and Nonlinear Control, 24, 228-246, 2014

[25] W. Perruquetti, J. P. Barbot, Sliding mode control in engineering, Marcel Dekker, Inc., 2002 